\title{
THE NUMBER HIDES GAME
}

\author{
V. J. BASTON, F. A. BOSTOCK AND T. S. FERGUSON
}

(Communicated by William O. Sudderth)

\begin{abstract}
We solve the following two-person zero-sum game, introduced by Ruckle. Players I and II simultaneously choose sequences of $m$ and $n$ consecutive integers respectively from the integers 1 to $p$ inclusive. The payoff to $\mathrm{I}$ is the number of integers in the intersection of the two sequences. A continuous version of this game is also solved as well as the variations in which one of the players need not choose his integers consecutively.
\end{abstract}

\section{INTRODUCTION AND SUMMARY}

The book by W. H. Ruckle, [2], contains a large number of geometric games and their applications, plus many unsolved problems. One of the unsolved games is called the "Number Hides Game" (NHG) and may be described as follows. The board, $C$, consists of the consecutive integers from 1 to $p, C=$ $\{1,2, \ldots, p\}$. Player I (Red) chooses a subset, $R$, of $m$ consecutive integers from $C$; simultaneously, Player II (Blue) chooses a subset, $B$, of $n$ consecutive integers from $C$. Then player II pays player I the number of elements in the intersection, $R \cap B$. This game is determined by the three integers, $m, n$, and $p$, where $1 \leq m \leq p$, and $1 \leq n \leq p$. The purpose of this paper is to present a solution to this game, denoted by $G(m, n, p)$.

This game is also described in [1] where it is called the "Number Search Game" (NSG), and a prize is offered for its solution. In these two references, certain special cases are solved.

Theorem 1 (Ruckle [1], [2]). If both $m$ and $n$ divide $p$, then the value of $G(m, n, p)$ is $m n / p$. An optimal strategy for I is the mixed strategy that chooses one of the intervals $\{1, \ldots, m\},\{m+1, \ldots, 2 m\}, \ldots,\{p-m+1, \ldots, p\}$ with probability $m / p$ each, and an optimal strategy for II is the mixed strategy that chooses one of the intervals $\{1, \ldots, n\},\{n+1, \ldots, 2 n\}, \ldots,\{p-n+1, \ldots, p\}$ with probability $n / p$ each.

The special case, $m=5, n=7, p=17$, is chosen in [2] to illustrate that the general solution must be more complex. The solution of $G(5,7,17)$ is stated as

Received by the editors January 20, 1988 and, in revised forms, August 26, 1988 and December 5, 1988.

1980 Mathematics Subject Classification (1985 Revision). Primary 90D05.

Key words and phrases. Two-person game, zero-sum game, geometric game. 
follows. The value is $7 / 3$; optimal for $I$ is to choose the sequence $\{3,4,5,6,7\}$ or $\{11,12,13,14,15\}$ with probability $1 / 3$ each or the sequence $\{4,5,6,7,8\}$ or $\{10,11,12,13,14\}$ with probability $1 / 6$ each; optimal for II is to choose the sequence $\{1,2,3,4,5,6,7\}$ or $\{11,12,13,14,15,16,17\}$ with probability $1 / 3$ each or the sequence $\{5,6,7,8,9,10,11\}$ or $\{7,8,9,10,11,12,13\}$ with probability $1 / 6$ each.

In section 2, the notation is set and some preliminary propositions are proved. In Proposition 2, it is noted that the games $G(m, n, p)$ and $G(n, m, p-n+m)$ are closely related. They have the same value and optimal strategies in that one can easily be transformed into optimal strategies in the other. This allows us to restrict attention to the case where $m \geq n$. Proposition 3 is a simple extension of Theorem 1 to a more general case using the same type of proof as used by Ruckle. In section 3, the general case is solved completely. In section 4, we present a solution to the continuous version of this game, called the Interval Overlap Game in Ruckle [2], and in doing so correct an error in the solution presented by Ruckle.

If both players are allowed to choose arbitrary sets of integers, $m$ for player I and $n$ for player II from the set $C=\{1, \ldots, p\}$, the game is called the Simple Point Catcher Game (SPCG) and solved in [2]. The value of the game is $m n / p$, and player I (resp. II) has an optimal strategy of choosing the set of $m$ (resp. $n$ ) integers from $C$ by simple random sampling (all subsets of $m$ (resp. $n$ ) integers from $C$ are equally likely). In the final section, the number hides game, modified by allowing one of the players to choose an arbitrary set of $m$ (or $n$ ) integers, is solved and given an interpretation in terms of minesweeping or linear Salvo.

\section{NOTATION AND PRELIMINARY RESULTS}

In the game $G(m, n, p)$, I has $p-m+1$ pure strategies and II has $p-n+1$ pure strategies. We number I's pure strategies from 0 to $p-m$, where strategy $i$ represents the choice $R=\{i+1, \ldots, i+m\}$. Similarly, II's pure strategies are numbered from 0 to $p-n$, where strategy $j$ represents the choice $B=\{j+$ $1, \ldots, j+n\}$. The payoff matrix of the game is therefore a $(p-m+1) \times(p-n+1)$ matrix, $A$, with components $a(i, j), i=0, \ldots, p-m, j=0, \ldots, p-n$, of the following form.

For $m \geq n$,

$$
\begin{array}{lll}
0 & \text { if } & 0 \leq j \leq i-n, \\
n-i+j & \text { if } & i-n \leq j \leq i \\
a(i, j)= & \text { if } & i \leq j \leq m+i-n, \\
m+i-j & \text { if } & m+i-n \leq j \leq m+i \\
0 & \text { if } & m+i \leq j \leq p .
\end{array}
$$


For $m \leq n$,

$$
a(i, j)=\begin{array}{llc}
0 & \text { if } & 0 \leq i \leq j-m, \\
m-j+i & \text { if } & j-m \leq i \leq j, \\
m & \text { if } & j \leq i \leq n+j-m, \\
n+j-i & \text { if } n+j-m \leq i \leq n+j, \\
0 & \text { if } & n+j \leq i \leq p .
\end{array}
$$

For example, the matrices of the games $G(3,5,7)$ and $G(3,5,10)$ are:

$$
G(3,5,7):\left(\begin{array}{ccc}
3 & 2 & 1 \\
3 & 3 & 2 \\
3 & 3 & 3 \\
2 & 3 & 3 \\
1 & 2 & 3
\end{array}\right) \quad G(3,5,10):\left(\begin{array}{llllll}
3 & 2 & 1 & 0 & 0 & 0 \\
3 & 3 & 2 & 1 & 0 & 0 \\
3 & 3 & 3 & 2 & 1 & 0 \\
2 & 3 & 3 & 3 & 2 & 1 \\
1 & 2 & 3 & 3 & 3 & 2 \\
0 & 1 & 2 & 3 & 3 & 3 \\
0 & 0 & 1 & 2 & 3 & 3 \\
0 & 0 & 0 & 1 & 2 & 3
\end{array}\right)
$$

A mixed strategy for $\mathrm{I}$ is a $p-m+1$ dimensional vector, say $\mathbf{r}=(r(0), r(1)$, $\ldots, r(p-m))$ with the understanding that $r(i)$ represents the probability that I chooses pure strategy $i$, namely $R=\{i+1, \ldots, i+m\}$. Similarly, a mixed strategy for II is a $p-n+1$ dimensional vector, say $\mathbf{b}=(b(0), b(1), \ldots, b(p-$ $n)$ ). Note that we start the numbering of these vectors as well as the matrix $A$ at 0 .

Our first task is to reduce the case $m \leq n$ to the case $m \geq n$. First we identify the cases of $m \leq n$ which have a saddle point, e.g. $G(3,5,7)$ above.

Proposition 1. If $m \leq n$ and $m+p \leq 2 n$, then $G(m, n, p)$ has a saddle point with value $m$. Any strategy is optimal for II, and the central row (or rows if $p-m$ is odd) is optimal for $\mathrm{I}$.

Proof. The conditions $m \leq n$ and $m+p \leq 2 n$ mean that $n$ is so large that II's choice is bound to contain the $m$ central integers of the set $C$.

If we observe the matrix for $G(3,5,10)$, we see that the first two rows are dominated by the third, and the last two rows are dominated by the third from last. When these four rows are deleted, the matrix becomes identical to the matrix of the game $G(5,3,8)$.

$$
G(5,3,8):\left(\begin{array}{llllll}
3 & 3 & 3 & 2 & 1 & 0 \\
2 & 3 & 3 & 3 & 2 & 1 \\
1 & 2 & 3 & 3 & 3 & 2 \\
0 & 1 & 2 & 3 & 3 & 3
\end{array}\right) .
$$

This means that the games $G(3,5,10)$ and $G(5,3,8)$ have the same value, and that any optimal strategy b for II in $G(3,5,10)$ is also optimal for II in $G(5,3,8)$ and conversely. Any strategy $\mathbf{r}$ optimal for $\mathrm{I}$ in $G(5,3,8)$ can be transformed into a strategy $\mathrm{r}^{\prime}$ optimal for $\mathrm{I}$ in $G(3,5,10)$ by adding two zeros to the front and end of $\mathbf{r}, \mathbf{r}^{\prime}=(0,0, r(0), \ldots, r(3), 0,0)$. Conversely, given any 
strategy $\mathbf{r}^{\prime}$ optimal for $\mathrm{I}$ in $G(3,5,10)$, combining the first three components of $\mathbf{r}^{\prime}$ into one and similarly the last three components gives a strategy $\mathbf{r}$ optimal in $G(5,3,8), \mathbf{r}=\left(r^{\prime}(0)+r^{\prime}(1)+r^{\prime}(2), r^{\prime}(3), r^{\prime}(4), r^{\prime}(5)+r^{\prime}(6)+r^{\prime}(7)\right)$. This observation is expressed in terms of arbitrary $m, n, p$ as follows.

Proposition 2. If $m \leq n$ and $m+p \geq 2 n$, then the games $G(m, n, p)$ and $G(n, m, p-n+m)$ are equivalent in the sense that the values are the same, player II's optimal strategies are the same and any strategy optimal for I in $G(n, m, p-n+m)$ is also optimal in $G(m, n, p)$ if padded in front and at the end by $n-m$ zeros.

Proof. It is sufficient to check first that the top and bottom $n-m$ rows of the game matrix for $G(m, n, p)$ are dominated (by the next top or bottom row), and second that component $a(i, j)$ of $G(n, m, p-n+m)$ from (2) is equal to the component $a(i+n-m, j)$ of $G(m, n, p)$ from (1). These computations are elementary and left to the reader.

The implication of this result is rather surprising. For example, in playing the game $G(3,5,10)$ the players could pretend to be playing $G(5,3,8)$. Thus, I pretends that he is choosing a sequence of length 5 and that his opponent is choosing a sequence of length 3 , provided that he also pretends that the board starts from the third integer.

We now assume that $m \geq n$. The next proposition also treats a special simple case. However, it generalizes Theorem 1 a great deal still using the same ideas in the proof.

Proposition 3. If $m \geq n$ and $p=M m+x$, where $n \leq x \leq m$, then the value of $G(m, n, p)$ is $n /(M+1)$. An optimal strategy for I is to choose one of the $M+1$ intervals $\{1, \ldots, m\},\{m+1, \ldots, 2 m\}, \ldots,\{(M-1) m+1, \ldots, M m\}$ and $\{p-m+1, \ldots, p\}$ with probability $1 /(M+1)$ each. An optimal strategy for II is to choose one of the $M+1$ intervals $\{1, \ldots, n\},\{m+1, \ldots, m+$ $n\}, \ldots,\{M m+1, \ldots, M m+n\}$ with probability $1 /(M+1)$ each.

Proof. If I uses the suggested strategy, then each integer is covered with probability at least $1 /(M+1)$, so the expected number of integers contained in any sequence of length $n$ chosen by II is at least $n /(M+1)$. If II uses the suggested strategy, then any interval of length $m$ chosen by $I$ is bound to overlap exactly $n$ of the integers receiving probability $1 /(M+1)$ from II's strategy, so that the expected number of integers in the overlap is $n /(M+1)$.

This result holds even if $\mathrm{II}$ is not restricted to choosing the $n$ integers to be consecutive. In addition, if we write the suggested optimal strategies in their vector form as $\mathbf{r}$ or $\mathbf{b}$, then both optimal strategies are independent of $n$ (provided $x \geq n$ ). It is useful to state as a corollary the corresponding result for $m \leq n$. It may be proved using Proposition 2 or directly as in Proposition 3.

Corollary 1. If $m \leq n$ and $p=N n+x$, where $0 \leq x \leq n-m$, then the value of $G(m, n, p)$ is $m / N$. An optimal strategy for I is to choose one of the $N$ 
intervals $\{n-m+1, \ldots, n\},\{2 n-m+1, \ldots, 2 n\}, \ldots,\{N n-m+1, \ldots, N n\}$ with probability $1 / N$ each. An optimal strategy for II is to choose one of the $N$ intervals $\{1, \ldots, n\},\{n+1, \ldots, 2 n\}, \ldots,\{(N-1) n+1, \ldots, N n\}$ with probability $1 / N$ each.

This corollary holds even if $I$ is not restricted to choosing consecutive integers. That these results contain Theorem 1 is easily seen if stated as follows.

Corollary 2. If $\max (m, n)$ divides $p$, then the value of $G(m, n, p)$ is $m n / p$. Proof. If $m \geq n$, take $x=m$ in Proposition 3. If $m \leq n$, take $x=0$ in Corollary 1 .

\section{The general CASE}

Suppose that $m$ and $n$ are fixed with $m \geq n$. Then Proposition 3 gives the value of $G(m, n, p)$ for various values of $p$, namely, $p=m, m+n \leq p \leq 2 m$, $2 m+n \leq p \leq 3 m, \ldots$, and it is seen that the value is constant on each of these intervals. The main theorem below shows that the value is linear on each of the remaining closed intervals, $m \leq p \leq m+n, 2 m \leq p \leq 2 m+n, \ldots$.

The optimal strategy of both players can be expressed in terms of the probability vector $\mathbf{q}(m, p)=\left(q_{j}(m, p), j=0,1, \ldots, p-m\right)$ defined as follows. If $p=M m$ for some $M \geq 1$, then

$$
q_{j}(m, p)=\begin{array}{ll}
1 / M & \text { for } j=0, m, 2 m, \ldots,(M-1) m, \\
0 & \text { otherwise. }
\end{array}
$$

If $p=M m+x$ for some $M \geq 1$ and $0<x<m$, then

$$
\begin{array}{ll}
(M-i) /(M(M+1)) & \text { for } j=i m, i=0,1, \ldots, M-1, \\
q_{j}(m, p)=i /(M(M+1)) & \text { for } j=i m+x, i=1,2, \ldots, M \\
0 & \text { otherwise. }
\end{array}
$$

Thus, for example, $\mathbf{q}(5,17)$ is the 13 dimensional vector $(3,0,0,1,0,2,0$, $0,2,0,1,0,0,3) / 12$. For $x \neq 0$, the vector $M(M+1) \mathbf{q}(m, p)$ is symmetric, of length $p-m+1$, has a decreasing sequence of integers at every $m$ th place starting with $M$ at the first place, and has an increasing sequence of integers every $m$ th place ending with $M$ in the last place.

Theorem 2. Suppose $m \geq n$ and $p=M m+x$ for some $M \geq 1$ and $0 \leq x \leq m$. Then, the value, $V(m, n, p)$, of $G(m, n, p)$ is

$$
V(m, n, p)=\begin{array}{ll}
(n(M+1)-x) /(M(M+1)) & \text { for } 0 \leq x \leq n, \\
n /(M+1) & \text { for } n \leq x \leq m .
\end{array}
$$

Moreover, $\mathbf{q}(m, p)$ is optimal for I and $\mathbf{q}(m, p+m-n)$ is optimal for II.

Remarks. The case $n \leq x \leq m$ is also covered in Proposition 3, but different optimal strategies are suggested for the players (except for player I when $x=m$, and for player II when $x=n$ ). However, one can show that the strategies proposed in Theorem 2 dominate those proposed in Proposition 3 (when they are different) in the sense that they never give a poorer expected return against 
any strategy of the opponent, and against some pure strategy of the opponent they give a strictly better return. In other words, the strategies of Theorem 2 are better suited to taking advantage of the opponent's mistakes.

As an example, let us solve the game $G(5,7,17)$. According to Proposition 3, $G(5,7,17)$ is equivalent to $G(7,5,15)$. From Theorem 2 , since $15=2 * 7+1$ (so $M=2$ and $x=1$ ), the value of $G(7,5,15)$ is $(5 * 3-1) /(2 * 3)=14 / 6=$ 7/3. Optimal for $I$ is

$$
\mathbf{r}=\mathbf{q}(7,15)=(2,1,0,0,0,0,0,1,2) / 6,
$$

and optimal for II is

$$
\mathbf{b}=\mathbf{q}(7,17)=(2,0,0,1,0,0,0,1,0,0,2) / 6 .
$$

Hence, $G(5,7,17)$ has the same value, $7 / 3$, player II has the same optimal strategy, b, and player I has the optimal strategy,

$$
(0,0, \mathbf{r}, 0,0)=(0,0,2,1,0,0,0,0,0,1,2,0,0) / 6 \text {. }
$$

This is the value and optimal strategy for I found in [2] and mentioned in the introduction. However, the optimal strategy for II found there is

$$
\mathbf{b}^{\prime}=(2,0,0,0,1,0,1,0,0,0,2) / 6 .
$$

Both $\mathbf{b}$ and $\mathbf{b}^{\prime}$ are optimal for II in both $G(7,5,15)$ and $G(5,7,17)$, but b dominates $\mathbf{b}^{\prime}$ in $G(7,5,15)$; it takes better advantage of any mistake player I may make by giving any weight to pure strategies $i=3,4,5,6,7$. In $G(5,7,17)$, neither $\mathbf{b}$ nor $\mathbf{b}^{\prime}$ dominates the other; $\mathbf{b}^{\prime}$ is better than $\mathbf{b}$ if $\mathbf{I}$ errs by using $i=1,2,12$ or 13 , while the reverse is true if I errs by using $5,6,7,8$ or 9 .

We state as a corollary the result corresponding to the case $m \leq n$, using the results of Proposition 2. As with Theorem 2, the value is seen to be constant on the intervals of Corollary 1 and linear between them.

Corollary 3. Suppose $m \leq n$ and $p=N n+x$ for some $N \geq 1$ and $0 \leq x \leq n$. Then,

$$
V(m, n, p)=\begin{array}{ll}
m / N & \text { for } \quad 0 \leq x \leq n-m, \\
(m N+n-x) /(N(N+1)) & \text { for } n-m \leq x \leq n
\end{array}
$$

Moreover, $\mathbf{q}(n, p)$ is optimal for II and $(\mathbf{0}, \mathbf{q}(n, p-n+m), \mathbf{0})$ is optimal for I, where 0 represents an $n-m$ dimensional vector of zeros.

It is interesting to note that the player who chooses the larger interval has an optimal strategy that is independent of the length of the smaller interval. However, unlike for Proposition 3, the general results require that the player choosing the smaller interval be restricted to choosing consecutive integers.

Proof of Theorem 2. Suppose that I uses $\mathbf{q}(m, p)$. If $p=M m$ for some $M \geq 1$, then Proposition 3 implies that $\mathrm{I}$ is guaranteed a return of at least $n /(M+1)$. If $p=M m+x$ with $M \geq 1$ and $0<x<m$, then the probability that square $s$ is covered by the interval chosen by $\mathbf{q}(m, p)$ is: 
(i) If $s=i m+y$ with $1 \leq y \leq x$,

$$
\operatorname{Prob}(s \text { covered })=[(M-i)+i] /(M(M+1))=1 /(M+1) \text {. }
$$

(ii) If $s=i m+y$ with $x+1 \leq y \leq m$,

$$
\operatorname{Prob}(s \text { covered })=[(M-i)+(i+1)] /(M(M+1))=1 / M \text {. }
$$

If II chooses an interval $R$ of length $n$, the expected payoff to I is the sum of these probabilities for $s$ in $R$. If $n \leq x$, the best II can do against this strategy is to choose $R$ entirely within one of the intervals of type $\mathrm{i}$ ), giving I an expected payoff of $n /(M+1)$. If $n>x$, the best II can do is to choose $R$ spanning one of the intervals of type i) with an overlap of $n-x$ into type ii), giving I an expected payoff of $x /(M+1)+(n-x) / M=[n(M+1)-x] /(M(M+1))$, as claimed.

Now suppose that II uses $\mathbf{q}(m, p+m-n)$ in $G(m, n, p)$ where $p=M m+x$, $0 \leq x \leq m$. We must distinguish several cases.

Case 1. $x=n$. In this case $p+m-n$ is divisible by $m$ and the strategy $\mathbf{q}(m, p+m-n)$ has the form (3). This strategy gives weight $1 /(M+1)$ to $s$ in the intervals $i m+1 \leq s \leq i m+n$ for $i=0,1, \ldots, M$, and weight 0 to other $s$. For any choice by $\mathrm{I}$ of the interval $R$ of length $n$, the sum of the weights is $n /(M+1)$, so this guarantees II an expected loss of $n /(M+1)$ as claimed.

Case 2. $x<n$ and $n \leq m-n+x$. The strategy $\mathbf{q}(m, p+m-n)$ is of the form (4). Let us define the weights given to a square $s$ as the probability given to $s$ times $M(M+1)$. Then letting $z$ denote $m-n+x$, the weights given to the board $C$ have the following pattern.

\begin{tabular}{|c|c|c|c|c|c|c|c|c|c|c|}
\hline & & $x$ & $n$ & $z$ & $m$ & & & & $2 m$ & \\
\hline weight & $M$ & $M$ & 1 & 1 & | $M$ & $|M-1|$ & 0 & 1 & $|M|$ & $\ldots$ \\
\hline 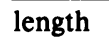 & $x$ & $n-$ & $z-n$ & $n-x$ & $x$ & $n-x$ & $z-n$ & $n-x$ & $x$ & \\
\hline
\end{tabular}

$$
\begin{array}{cc}
\text { interval } & \text { weight } \\
i m+1 \leq s \leq i m+x & M \\
i m+x+1 \leq s \leq i m+n & M+1-i \\
i m+n+1 \leq s \leq i m+z & 0 \\
i m+z+1 \leq s \leq i m+m & i-1
\end{array}
$$

From this, we can compute the total weight given to any interval of length $m$ chosen by $I$. If I chooses the interval $\{1, \ldots, m\}$, the total weight is $M x+$ $M(n-x)+(n-x)=(M+1) n-x$. Since this divided by $M(M+1)$ is the claimed optimal value, we must show that no other choice of an interval by I can give him a greater total weight. Consider what happens as I advances his chosen interval. As the starting integer advances through $x$, the total weight stays the same since as a weight $M$ is removed a weight $M$ is added. As we proceed farther through $n$, a weight $M$ is removed and a weight $M-1$ is added so the total weight goes down by 1 at each step. Then it stays constant through $z$, and then it increases 1 at each step through $m$. The total weight has returned 
to $(M+1) n-x$ since it decreased 1 for $n-x$ steps and increased 1 for $n-x$ steps. This procedure clearly continues through the next $m$ steps giving the same sequence of total weights. Thus, no matter where I chooses the interval of length $m$, his expected payoff is never greater than $[(M+1) n-x] /(M(M+1))$, as was to be shown.

Case 3. $x<n$ and $n>m-n+x$. A similar analysis can be given in the remaining cases. We just specify the pattern of the weights. If II uses $\mathbf{q}(m, p+$ $m-n$ ), then the weights have the following pattern, (again $z=m-n+x$ ).

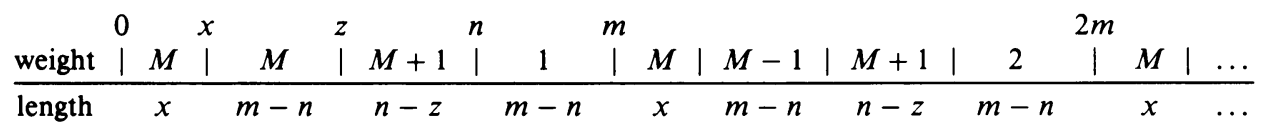

If I chooses the interval $\{1, \ldots, m\}$, the total weight is $M z+(M+1)(n-$ $z)+(m-n)=(M+1) n-x$. As the starting point increases, the total weight stays constant through $x$, decreases through $z$, stays constant through $n$ and increases through $m$ back to its original value. Since this repeats with a period of $m$, the greatest expected payoff I can receive is $[(M+1) n-x] /(M(M+1))$, as claimed.

Case 4. $x>n$ and $n \leq x-n$. Since $p+m-n=(M+1) m+(x-n)$, the strategy $\mathbf{q}(m, p+m-n)$ is of the form (4) with $M$ replaced by $M+1$ and $x$ replaced by $x-n$. The weights, here defined as the probabilities times $(M+1)(M+2)$, have the following pattern, where $z$ now denotes $x-n$.

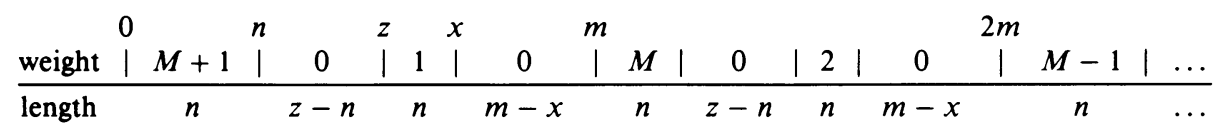

If I chooses the interval $\{1, \ldots, m\}$, the total weight is $(M+1) n+n=$ $(M+2) n$. As the starting point increases, the total weight decreases through $n$, stays constant through $z$, increases through $x$, and stays constant through $m$ at its original value. Since this repeats with a period of $m$, the greatest expected payoff I can receive is $(M+2) n /((M+1)(M+2))=n /(M+1)$, as claimed.

Case 5. $x>n$ and $n>x-n$. With the weights and $z$ as defined in Case 4, the pattern of the weights is as follows.

\begin{tabular}{|c|c|c|c|c|c|c|c|c|c|c|c|}
\hline & & & $n$ & $x$ & $m$ & & & & $2 m$ & & \\
\hline weight & $M+1$ & $M+2$ & 1 & | & | $M$ & $M+2$ & 21 & 0 & 1 & $M-1$ & $\ldots$ \\
\hline ength & $z$ & $n-z$ & $z$ & $m-x$ & $z$ & $n-z$ & $z$ & $m-x$ & & $z$ & \\
\hline
\end{tabular}

If I chooses the interval $\{1, \ldots, m\}$, the total weight is $(M+1) z+(M+$ $2)(n-z)+z=(M+2) n$. As above, this is the greatest weight achievable by $\mathrm{I}$, giving him an expected payoff of $n /(M+1)$, as claimed. 


\section{THE INTERVAL OVERLAP GAME}

The Interval Overlap Game is the continuous analog of the Number Hides Game. I and II simultaneously choose subintervals $R$ and $B$ of the interval $[0,1]$ of fixed lengths $\alpha$ and $\beta$ respectively, and the payoff to $I$ is the length of the intersection, $R \cap B$. The ideas developed in the previous sections carry over to this game and the reader should have little difficulty convincing himself that the following theorem holds.

Theorem 3. Let $M$ (resp. $N$ ) be the greatest integer less than or equal to $1 / \alpha$ (resp. 1/B). The value of the Interval Overlap Game is

$$
\begin{array}{cl}
\beta /(M+1) & \text { if } \alpha \geq \beta \text { and } \beta \leq 1-M \alpha, \\
(M \alpha+M \beta+\beta-1) /(M(M+1)) & \text { if } \alpha \geq \beta \text { and } \beta \geq 1-M \alpha, \\
\alpha / N & \text { if } \alpha \leq \beta \text { and } 1+\alpha \leq N \beta+\beta, \\
(N \alpha+N \beta+\beta-1) /(N(N+1)) & \text { if } \alpha \leq \beta \text { and } 1+\alpha \geq N \beta+\beta .
\end{array}
$$

When $\alpha \geq \beta$, I has an optimal strategy whereby, for $k=0,1, \ldots, M-1$, he chooses $[k \alpha,(k+1) \alpha]$ with probability $(M-k) /(M(M+1))$ and $[1-(M-$ $k) \alpha, 1-(M-k-1) \alpha]$ with probability $(k+1) /(M(M+1))$.

When $\alpha \geq \beta$, II has an optimal strategy whereby, for $k=0,1, \ldots, M-1$, he chooses $[k \alpha, k \alpha+\beta]$ with probability $(M-k) /(M(M+1))$ and $[1-\beta-$ $(M-k-1) \alpha, 1-(M-k-1) \alpha]$ with probability $(k+1) /(M(M+1))$.

When $\alpha \leq \beta$, I has an optimal strategy whereby, for $k=0,1, \ldots, N-1$, he chooses $[(k+1) \beta-\alpha,(k+1) \beta]$ with probability $(N-k) /(N(N+1))$ and $[1-(N-k) \beta, 1-(N-k) \beta+\alpha]$ with probability $(k+1) /(N(N+1))$.

When $\alpha \leq \beta$, II has an optimal strategy whereby, for $k=0,1, \ldots, N-1$, he chooses $[k \beta,(k+1) \beta]$ with probability $(N-k) /(N(N+1))$ and $[1-(N-$ $k) \beta, 1-(N-k) \beta+\beta$ ] with probability $(k+1) /(N(N+1))$.

Ruckle investigated the Interval Overlap Game in [2, pg. 77], but unfortunately his analysis is not totally correct concerning optimal strategies for II. For instance, his analysis implies that an optimal strategy for II in the case $\alpha=.4$ and $\beta=.35$ is to choose one of the intervals $[0,14 / 40],[13 / 40,27 / 40]$, $[26 / 40,1]$ with probability $1 / 3$ each. However if I chooses the interval $[12 / 40$, $28 / 40$ ], he can expect at least $(1 / 3)(2 / 40)+(1 / 3)(14 / 40)+(1 / 3)(2 / 40)=$ $18 / 120$ which is greater than the value of the game. It can be seen that similar considerations apply for his analysis of the case $\alpha<\beta$ by looking at the special case $\alpha=.39$ and $\beta=.4$.

\section{MODIFIED GAMES}

In this section, the rules of the game are changed to allow one of the players to choose an arbitrary set of integers not necessarily consecutive.

First, we suppose that player II is allowed to choose an arbitrary set of $n$ integers from $C$, while player I is still restricted to choosing a set of $m$ consecutive integers. The payoff is still the number of integers in the intersection 
of the chosen sets. Let us denote this game by $G_{1}(m, n, p)$. It will be seen that the strategy $\mathbf{q}(m, p)$ still plays a role in the analysis.

In keeping with Ruckle's tradition of presenting applications, we give the following interpretation of this game. Player II is seeking to disrupt a busy channel of water having $p$ shipping lanes and has mines which he can distribute in $n$ of the lanes. Player I wants to keep the channel clear for shipping but his only resource is a minesweeper that can sweep just $m$ consecutive lanes; he wins the number of mines that he finds.

Theorem 4. If $p=M m+x$ where $0 \leq x \leq m$ and $M \geq 1$, then the value of $G_{1}(m, n, p)$ is

$$
V_{1}(m, n, p)=\begin{array}{ll}
(n-x) / M & \text { if } \quad 0 \leq x \leq n /(M+1) \\
n /(M+1) & \text { if } n /(M+1) \leq x \leq m
\end{array}
$$

Moreover, $\mathbf{q}(m, p)$ is optimal for I. Optimal for II is the following strategy. Let $S=\bigcup_{j=0}^{M}\{s \mid j m+1 \leq s \leq j m+x\}$. If $n /(M+1) \leq x \leq m$, choose all $n$ points from $S$ by simple random sampling. If $0 \leq x \leq n /(M+1)$, choose all $(M+1) x$ points of $S$, and choose the remaining $n-(M+1) x$ points from $C-S$ by simple random sampling.

Proof. Suppose I uses $\mathbf{q}(m, p)$. As in the proof of Theorem 2, the probability that a point $s \in C$ is covered by the interval chosen by $\mathrm{I}$ is

$$
\operatorname{Prob}(s \text { covered })=\begin{array}{ll}
1 / M & \text { if } s \in S, \\
1 /(M+1) & \text { if } s \in C-S .
\end{array}
$$

Against this strategy, II should choose as many points as possible in $S$, and the remaining in $C-S$. If $(M+1) x \geq n$, then II can choose all $n$ points in $S$ and so hold the expected payoff to $n /(M+1)$. If $(M+1) x \leq n$, then II can choose all $(M+1) x$ points of $S$ plus any $n-(M+1) x$ points of $C-S$ and so hold the expected payoff to $(M+1) x / M+(n-(M+1) x) /(M+1)=(n-x) / M$, as claimed.

Now suppose II uses the strategy stated in the theorem. If $n /(M+1) \leq x \leq$ $m$, then since any interval chosen by I contains exactly $x$ elements of $S$, the expected payoff to $I$ is exactly $x$ times the probability that an arbitrary point of $S$ is chosen by II, namely, $x n /((M+1) x)=n /(M+1)$, no matter what interval I chooses. If $0 \leq x \leq n /(M+1)$, then similarly, the expected payoff to $\mathrm{I}$ is exactly $x+(m-x)(n-(M+1) x) /(M(m-x))=(n-x) / M$, no matter what interval I chooses, as claimed.

An analysis similar to the above can be carried out on the game $G_{2}(m, n, p)$, in which $\mathrm{I}$ is allowed to choose any $m$ points in $C$ while II is restricted to choosing $n$ consecutive integers, the payoff again being the number of points in the intersection. We can think of $G_{2}(m, n, p)$ as the game of Salvo, or Battleship played on a line. Player II hides a battleship of length $n$ on a line of length $p$ vertices, and Player I chooses $m$ spots for his salvo, winning the number of hits. We state the result without proof. 
Theorem 5. If $p=N n+x$ where $0 \leq x \leq n$ and $N \geq 1$, then the value of $G_{2}(m, n, p)$ is

$$
V_{2}(m, n, p)=\begin{array}{ll}
(m+n-x) /(N+1) & \text { if } 0 \leq n-x \leq m / N, \\
m / N & \text { if } m / N \leq n-x \leq n .
\end{array}
$$

Moreover, $\mathbf{q}(n, p)$ is optimal for II. Optimal for I is the following strategy. Let $S=\bigcup_{j=1}^{N}\{s \mid(j-1) n+x+1 \leq s \leq j n\}$. If $m / N \leq n-x \leq n$, choose all $m$ points from $S$ by simple random sampling. If $0 \leq n-x \leq m / N$, choose the $N(n-x)$ points of $S$, and choose the remaining $m-N(n-x)$ points from $C-S$ by simple random sampling.

\section{REFERENCES}

1. W. H. Ruckle, Technical Report \#384, Department of Mathematical Sciences, Clemson University, 1982.

2. _ Geometric games and their applications, Research Notes in Mathematics No. 82, Pitman Advanced Publishing Program, Boston, London, Melbourne, 1983.

(V. J. Baston and F. A. Bostock): Faculty of Mathematical Studies, University of Southampton, Southampton S09 5NH, UNITEd KINGdOM

(T. S. Ferguson): Mathematics Department, University of California at Los Angeles, Los ANGeles, CALIFornia 90024 